Article

\title{
3D hierarchically macro-/mesoporous graphene frameworks enriched with pyridinic-nitrogen-cobalt active sites as efficient reversible oxygen electrocatalysts for rechargeable zinc-air batteries
}

\author{
Sheng Zhou, Jiayi Qin, Xueru Zhao, Jing Yang * \\ Institute of New-Energy Materials, Key Laboratory of Advanced Ceramics and Machining Technology of Ministry of Education, School of Materials Science \\ and Engineering, Tianjin University, Tianjin 300072, China
}

\section{A R T I C L E I N F O}

\section{Article history:}

Received 27 April 2020

Accepted 31 May 2020

Available online 5 September 2020

\section{Keywords:}

Hierarchical pores

Composite catalyst

Oxygen electrocatalysis

Spinel oxide

Rechargeable zinc-air battery

\begin{abstract}
A B S T R A C T
Efficient and affordable electrocatalysts for reversible oxygen reduction and oxygen evolution reactions (ORR and OER, respectively) are highly sought-after for use in rechargeable metal-air batteries. However, the construction of high-performance electrocatalysts that possess both largely accessible active sites and superior ORR/OER intrinsic activities is challenging. Herein, we report the design and successful preparation of a 3D hierarchically porous graphene framework with interconnected interlayer macropores and in-plane mesopores, enriched with pyridinic-nitrogen-cobalt (pyri-N-Co) active sites, namely, CoFe/3D-NLG. The pyri-N-Co bonding significantly accelerates sluggish oxygen electrocatalysis kinetics, in turn substantially improving the intrinsic ORR/OER activities per active site, while copious interlayer macropores and in-plane mesopores enable ultra-efficient mass transfer throughout the graphene architecture, thus ensuring sufficient exposure of accessible pyri-N-Co active sites to the reagents. Such a robust catalyst structure endows $\mathrm{CoFe} / 3 \mathrm{D}-\mathrm{NLG}$ with a remarkably enhanced reversible oxygen electrocatalysis performance, with the ORR half-wave potential identical to that of the benchmark Pt/C catalyst, and OER activity far surpassing that of the noble-metal-based $\mathrm{RuO}_{2}$ catalyst. Moreover, when employed as an air electrode for a rechargeable $\mathrm{Zn}$-air battery, $\mathrm{CoFe} / 3 \mathrm{D}-\mathrm{NLG}$ manifests an exceedingly high open-circuit voltage ( $1.56 \mathrm{~V})$, high peak power density $(213 \mathrm{~mW} \mathrm{~cm}-2)$, ultra-low charge/discharge voltage $(0.63$ V), and excellent charge/discharge cycling stability, outperforming state-of-the-art noble-metal electrocatalysts.
\end{abstract}

(C) 2021, Dalian Institute of Chemical Physics, Chinese Academy of Sciences. Published by Elsevier B.V. All rights reserved.

\section{Introduction}

With the energy crisis and environmental pollution increasing in severity, sustainable electrochemical energy storage and conversion technologies have attracted considerable attention and research interests [1]. Reversible oxygen electrocatalysis, including oxygen reduction and oxygen evolution reac- tions (ORR and OER, respectively), is essential for air cathodes in rechargeable metal-air batteries; however, these processes are often hindered by sluggish kinetics and high overpotentials, due to complicated multi-electron transfer steps [2]. Currently, $\mathrm{Pt} / \mathrm{C}$ and $\mathrm{RuO}_{2}$ (or $\mathrm{Ir} / \mathrm{C}$ and $\mathrm{IrO}_{2}$ ) are considered as benchmark electrocatalysts for ORR and OER, respectively [3]; unfortunately, they lack efficiency as bifunctional catalysts for reversi-

* Corresponding author. Tel: +86-22-85356663; E-mail: yang_jing@tju.edu.cn 
ble ORR/OER. Additionally, the prohibitive cost, scarcity, and poor durability of noble-metal catalysts severely hinder their large-scale industry implementation [4]. Therefore, efficient and affordable bifunctional ORR/OER electrocatalysts are highly desirable for high-performance rechargeable metal-air batteries.

3D-transition-metal oxides nanoparticles (TMO NPs) anchored on nitrogen-doped carbonaceous supports are regarded as highly promising electrocatalysts for reversible oxygen electrode reactions, due to their high abundance, low cost, low toxicity, and attractive ORR/OER catalytic performance [5-9]. The favorable electrocatalytic activity has been attributed to the synergistic effect originating from the $\mathrm{M}-\mathrm{N}-\mathrm{C}(\mathrm{M}=\mathrm{Fe}, \mathrm{Co}, \mathrm{Cu}$, etc.) species at the composite interface [10-13]. Recently, our research further revealed the importance of pyridinic-nitrogen-cobalt (pyri-N-Co) bonding, as opposed to pyrrolic-N-Co bonding, for the synergistic effect, which substantially promoted the intrinsic ORR/OER activities of $\mathrm{Ni}$ $\mathrm{Co}_{2} \mathrm{O}_{4} / \mathrm{N}$-doped graphene [14]. Nevertheless, as a highly conductive support for TMO NPs, 2D graphene sheets are ordinarily unavoidably stacked face-to-face when loaded onto oxygen electrodes, resulting in a severe loss of accessible catalyst active sites in practical applications. Therefore, in order to optimize catalytic activities, catalyst design strategies should aim at achieving excellent intrinsic activity, as well as a large number of exposed active sites [15].

$3 \mathrm{D}$ porous graphene, with a large surface area, is capable of adequate exposure of active sites, and has been adopted as a catalyst support in numerous recent studies, as a means of improving catalytic activity [16-19]. It has been widely recognized that the interlayer macropores (> $100 \mathrm{~nm}$ ) between graphene sheets ensure rapid permeation and diffusion of liquid electrolyte and oxygen in catalytic processes [20-23]. On the contrary, in-plane mesopores $(2-50 \mathrm{~nm})$, present within individual graphene planes, have rarely been exploited in electrocatalysts, although they may be capable of entirely interconnecting the interlayer macropores, and thus providing favorable shortcuts for efficient ionic diffusion, as reported in recent studies on supercapacitors [24-26]. In this regard, hierarchically porous graphene composed of both interlayer macropores and in-plane mesopores could serve as an ideal catalyst support for electrocatalysis, not only ensuring favorable exposure of active sites accessible to the regents, but also enabling substantially improved mass transfer and charge transport [27]. However, studies regarding the combination of such a graphene architecture with abundant active sites for reversible oxygen electrocatalysis are scarce.

In this work, a 3D macro-/mesoporous $\mathrm{N}$-doped graphene framework loaded with $\mathrm{CoFe}_{2} \mathrm{O}_{4}$ NPs (namely, CoFe/3D-NLG) enriched with pyri-N-Co active sites, was designed and successfully fabricated via facile laser irradiation and hydrothermal methods; the material possesses excellent intrinsic ORR/OER activities at each active site, in addition to large amounts of readily accessed active sites. Specifically, the interlayer macropores arising from the self-assembly of graphene films benefit oxygen gas and electrolyte penetration. More importantly, the numerous laser-irradiation-induced in-plane mesopores are not only favorable for the formation of abundant highly efficient pyri-N-Co active sites for reversible ORR/OER, but also for further reducing the diffusion path lengths of reagents and products throughout the catalyst during catalytic processes. The interlayer macropores and in-plane mesopores together provide extensive exposure of the pyri-N-Co active sites to the reagents. Remarkably, the CoFe/3D-NLG electrocatalyst exhibited outstanding catalytic performances for both ORR and OER in alkaline conditions, with a very low potential difference between ORR and OER $(\Delta E$ $=0.67 \mathrm{~V}$ ), surpassing the majority of reported highly active Coand/or Fe-based bifunctional electrocatalysts, as well as a mixture of benchmark noble-metal catalysts $\left(\mathrm{Pt} / \mathrm{C}+\mathrm{RuO}_{2}\right)$. The CoFe/3D-NLG-driven rechargeable Zn-air battery demonstrates superior charge/discharge performance compared to the noble-metal based catalysts.

\section{Experimental}

\subsection{Materials}

All reagents used in the experiments were of analytical grade and were used without further purification. The graphite powder (325 mesh) was obtained from Sigma-Aldrich. Sulfuric acid $\left(\mathrm{H}_{2} \mathrm{SO}_{4}\right)$, potassium thiosulfate $\left(\mathrm{K}_{2} \mathrm{~S}_{2} \mathrm{O}_{8}\right)$, phosphorus pentoxide $\left(\mathrm{P}_{2} \mathrm{O}_{5}\right)$, acidic potassium permanganate $\left(\mathrm{K}_{2} \mathrm{MnO}_{4}\right)$, ethanol $\left(\mathrm{CH}_{3} \mathrm{CHOH}\right)$, and hydrochloric acid $(\mathrm{HCl})$ were obtained from Tianjin Jiangtian Chemicals. 25\% hydrogen peroxide $\left(\mathrm{H}_{2} \mathrm{O}_{2}\right)$ were gained from Reagent Chemicals. Ammonia $\left(\mathrm{NH}_{3} \cdot \mathrm{H}_{2} \mathrm{O}\right)$ was bought from Kermel. Cobalt chloride hexahydrate $\left(\mathrm{CoCl}_{2} \cdot 6 \mathrm{H}_{2} \mathrm{O}\right)$ and Iron(II) chloride tetrahydrate $\left(\mathrm{FeCl}_{2} \cdot 4 \mathrm{H}_{2} \mathrm{O}\right)$ were obtained from Macklin. Nafion solution (5 wt\%), $\mathrm{KOH}$ (99.98\%), Pt/C (20 wt\%), and $\mathrm{RuO}_{2}$ were obtained from Tianjin Incole Union Technology Co., Ltd

\subsection{Electrocatalyst preparation}

Synthesis of graphene oxide (GO). GO was prepared from graphite powder using a modified Hummer method [28]. Briefly, in the pre-oxidation process, $2.5 \mathrm{~g} \mathrm{P}_{2} \mathrm{O}_{5}$ and $2.5 \mathrm{~g} \mathrm{~K}_{2} \mathrm{~S}_{2} \mathrm{O}_{8}$ were dissolved into $15 \mathrm{~mL}$ of $\mathrm{H}_{2} \mathrm{SO}_{4}$. Here, $\mathrm{P}_{2} \mathrm{O}_{5}$ was used to produce intact graphitic basal planes, $\mathrm{K}_{2} \mathrm{~S}_{2} \mathrm{O}_{8}$ was adopted to shorten the oxidation time of graphite powder and improve the extent of oxidation, and $\mathrm{H}_{2} \mathrm{SO}_{4}$ acted as intercalation reagents of graphite. Then $5 \mathrm{~g}$ graphite powder was added, while keeping stirring. The obtained solution was heated at $80{ }^{\circ} \mathrm{C}$ for $6 \mathrm{~h}$. When the solution temperature dropped to room temperature, $500 \mathrm{~mL}$ of $\mathrm{H}_{2} \mathrm{O}$ was added to dilute the solution. The pre-oxidized graphene was obtained by centrifugation, then rinsed for several times with pure water, and dried at $50{ }^{\circ} \mathrm{C}$ in an oven. The next step is oxidation, where the pre-oxidized graphene was dispersed into $120 \mathrm{~mL}$ of $\mathrm{H}_{2} \mathrm{SO}_{4}$ at $0{ }^{\circ} \mathrm{C}$ and stirred for $10 \mathrm{~min}$. Then, $17.5 \mathrm{~g} \mathrm{KMnO}_{4}$, which could oxidize the acid-intercalated graphite and result in graphite oxides, was slowly added into the suspension, while the temperature was kept between 10 and $15^{\circ} \mathrm{C}$. The suspension was then kept stirring at $35{ }^{\circ} \mathrm{C}$ for $2 \mathrm{~h}$. Next, $250 \mathrm{~mL}$ of $\mathrm{H}_{2} \mathrm{O}$ was added to dilute 
the solution in ice bath, and the temperature was set to be below $50{ }^{\circ} \mathrm{C}$ during the process, while keep stirring for another 2 h. Then $750 \mathrm{~mL}$ of $\mathrm{H}_{2} \mathrm{O}$ was added to the solution. Finally, 20 $\mathrm{mL}$ of hydrogen peroxide $\left(\mathrm{H}_{2} \mathrm{O}_{2}\right)$ solution was added, leading to a gradual color change of the solution to orange with the formation of bubbles. Then $1 \mathrm{~L}$ of $0.1 \mathrm{M}$ hydrochloric acid ( $\mathrm{HCl}$ ) was added while keeping stirring for $45 \mathrm{~min}$. The GO was collected by centrifugation, rinsed with pure water for many times until $\mathrm{pH}=7$, and then dried by lyophilization.

Synthesis of graphene oxide sheets with in-plane mesoporous (LGO). $100 \mathrm{mg} \mathrm{GO}$ was dispersed in $300 \mathrm{~mL}$ of ethanol to make a suspension via ultrasonication. The resultant suspension was irradiated by an unfocused nanosecond pulsed $\mathrm{Nd}$ :YAG laser (1064 nm, $7 \mathrm{~ns}, 15 \mathrm{~Hz}$ ) in an ice water bath, without continuous stirring. The laser pulse energy is about $270 \mathrm{~mJ}$, and the irradiation time is $30 \mathrm{~min}$. The precipitate was collected by centrifugation and dried by lyophilization. The energy dispersive spectra of GO and LGO (Fig. S1) confirm that only $\mathrm{C}$ and $\mathrm{O}$ are present in GO and LGO.

Synthesis of 2D N-doped mesoporous graphene sheets supported $\mathrm{CoFe}_{2} \mathrm{O}_{4}$ nanoparticles (NPs) (CoFe/NLG). $15 \mathrm{mg}$ LGO was dispersed in $30 \mathrm{~mL}$ of $\mathrm{H}_{2} \mathrm{O}$ to make a suspension via ultrasonication. And then $100 \mathrm{uL}$ of $\mathrm{NH}_{3} \cdot \mathrm{H}_{2} \mathrm{O}$ and $100 \mathrm{mg}$ urea were added, and the mixture was transferred into an autoclave and heated at $180{ }^{\circ} \mathrm{C}$ for $12 \mathrm{~h}$. Then the autoclave was taken out to cool down to room temperature. Next, $106 \mathrm{mg} \mathrm{FeCl}_{2} \cdot 4 \mathrm{H}_{2} \mathrm{O}$ and $60 \mathrm{mg} \mathrm{CoCl} \cdot 6 \mathrm{H}_{2} \mathrm{O}$ were dissolved into the solution, and then annealed at $180{ }^{\circ} \mathrm{C}$ for $3 \mathrm{~h}$. The products were collected by centrifugation and dried by lyophilization. The preparation of $\mathrm{N}$-doped graphene sheets supported $\mathrm{CoFe}_{2} \mathrm{O}_{4} \mathrm{NPs}$ ( $\mathrm{CoFe} / \mathrm{NG}$ ) follows the same procedures as CoFe/NLG, except that LGO was replaced by GO.

Synthesis of 3D macro-/mesoporous N-doped graphene framework loaded with $\mathrm{CoFe}_{2} \mathrm{O}_{4} \mathrm{NPs}$ (CoFe/3D-NLG). $30 \mathrm{mg}$ LGO was dispersed in $15 \mathrm{~mL}$ of pure water to make a suspension via ultrasonication, and then $50 \mathrm{uL}$ of $\mathrm{NH}_{3} \cdot \mathrm{H}_{2} \mathrm{O}$ and $50 \mathrm{mg}$ urea were added. The mixture was transferred into an autoclave and heated at $180{ }^{\circ} \mathrm{C}$ for $12 \mathrm{~h}$. According to the literature [29], the assembly of GO in solution is greatly affected by its surface chemistry and the GO concentration should be higher than $1.0 \mathrm{mg} \mathrm{mL}^{-1}$. Therefore, in order to enable successful self-assembly of GO sheets, the GO concentration was set as 2.0 $\mathrm{mg} \mathrm{mL}^{-1}$ for preparing CoFe/3D-NLG, much higher than that for preparing 2D CoFe/NLG $\left(0.5 \mathrm{mg} \mathrm{mL}^{-1}\right)$. Besides, the amounts of ammonia, urea, and metal salts were adjusted accordingly. Then the metal stocks (71 mg $\mathrm{FeCl}_{2} \cdot 4 \mathrm{H}_{2} \mathrm{O}$ and $40 \mathrm{mg}$ $\mathrm{CoCl}_{2} \cdot 6 \mathrm{H}_{2} \mathrm{O}$ ) were added to make a suspension by ultrasonication, and heated at $180{ }^{\circ} \mathrm{C}$ for $3 \mathrm{~h}$ to allow the growth of $\mathrm{CoFe}_{2} \mathrm{O}_{4}$ nanoparticles on the surface of 3D-NLG. The as-obtained $\mathrm{CoFe} / 3 \mathrm{D}-\mathrm{NLG}$ were collected by centrifugation and dried by lyophilization. The preparation of 3D macroporous $\mathrm{N}$-doped graphene framework loaded with $\mathrm{CoFe}_{2} \mathrm{O}_{4} \mathrm{NPs}$ (i.e., $\mathrm{CoFe} / 3 \mathrm{D}-\mathrm{NG}$ ) follows similar procedures as CoFe/3D-NLG, except that LGO was replaced by GO.

\subsection{Characterization}

The scanning electron microscopy (SEM) images were carried out on a Hitachi S-4800 SEM system. Transmission electron microscopy (TEM), high-resolution TEM (HRTEM), energy dispersive spectroscopy (EDS), and electron energy loss spectroscopy (EELS) were acquired by using an FEI Tecnai G2 F20 transmission electron microscope equipped with a field-emission gun. The specific surface area was evaluated by the Brunauer-Emmett-Teller (BET) method, and the pore-size distribution was derived from the adsorption branch based on Quenched Solid Density Function Theory model. The powder X-ray diffraction (XRD) patterns of the samples were recorded on a Siemens-Bruker D5000 X-ray diffraction diffractometer with $\mathrm{Cu} K_{\alpha}$ radiation. Raman spectra were taken on a DXR Raman microscope at an excitation wavelength of $532 \mathrm{~nm}$. X-ray photoelectron spectroscopy (XPS) analyses were performed using a PHI Quantum 2000 scanning ESCA Microprobe spectrometer.

\subsection{Electrochemical measurements.}

Working Electrode Preparation. $2 \mathrm{mg}$ of as-prepared catalyst were ultrasonically dispersed in $390 \mathrm{uL}$ of deionized water, followed by the addition of $10 \mathrm{uL}$ of Nafion solution ( $5 \mathrm{wt} \%$ ) into the solution. The mixture was ultrasonicated to obtain a homogeneous catalyst ink. Certain amount of the catalyst ink were then transferred onto the glassy carbon rotating disk electrode (RDE, $0.196 \mathrm{~cm}^{2}$ ) or rotating ring-disk electrode (RRDE, $\left.0.246 \mathrm{~cm}^{2}\right)$ or carbon fiber paper $\left(0.25 \mathrm{~cm}^{2}\right)$ to ensure a constant catalyst mass loading of $0.4 \mathrm{mg} \mathrm{cm}^{-2}$ for all the measurements. The electrodes were then dried in air and served as a working electrode.

Electrochemical Tests. Most electrochemical tests were performed on a wave drive 20 workstation (Pine Research Instruments, US), except that OER, electrochemical impedance spectroscopy (EIS) and electrochemical surface area (ECSA) data were obtained on a $\mathrm{CHI} 600 \mathrm{E}$ electrochemical workstation. All of the electrochemical tests were carried out using a three-electrode system including a carbon rod counter electrode, a saturated calomel reference electrode, and a working electrode. The reference electrode was calibrated in $\mathrm{H}_{2}$-saturated 0.1 and $1 \mathrm{M} \mathrm{KOH}$ solution before measurements. For OER, hydrophilic carbon fiber paper (CFP) loaded with catalysts served as the working electrode, and tested in $\mathrm{O}_{2}$ saturated $1 \mathrm{M} \mathrm{KOH}$ electrolyte. For ORR, catalysts were loaded on a RDE or a RRDE as the working electrode, using an AF-MSRCE modulator speed rotator (Pine Instrument Inc.), and the ORR activity was measured in $\mathrm{O}_{2}$ saturated 0.1 or $1 \mathrm{M} \mathrm{KOH}$ electrolyte. All experiments were carried out at $25^{\circ} \mathrm{C}$.

Before data collection, cyclic voltammetry (CV) curves were repeatedly recorded at scan rate of $50 \mathrm{mV} \mathrm{s}^{-1}$ in a potential window of 1.05-1.85 V vs. RHE for OER or $0.2-1 \mathrm{~V}$ vs. RHE for ORR, until the signals were stabilized. Then linear sweep voltammograms (LSV) were measured at a scan rate of $5 \mathrm{mV} \mathrm{s}^{-1}$. The ORR polarization curves were recorded at different rotating speeds (i.e., 400, 625, 900, 1225, 1600, and $2025 \mathrm{rpm}$ ), and corrected by subtracting the background current for argon-saturated electrolyte. 
The ORR kinetics was evaluated by using the Koutechy-Levich (K-L) equation:

$$
\frac{1}{J}=\frac{1}{J_{\mathrm{d}}}+\frac{1}{J_{\mathrm{k}}}=\frac{1}{B \omega^{1 / 2}}+\frac{1}{J_{\mathrm{k}}}
$$

Where $J, J_{\mathrm{d}}$, and $J_{\mathrm{k}}$ represent the measured, diffusion-limiting, and kinetic current density, respectively. $B=0.2 n F A C_{0} D^{2 / 3} v^{-1 / 6}$, where $n$ is the electron transfer number, $F$ is the Faraday constant ( $\left.96485 \mathrm{C} \mathrm{mol}^{-1}\right), A$ is the electrode area $\left(0.196 \mathrm{~cm}^{2}\right), C_{0}$ is the saturated $\mathrm{O}_{2}$ concentration $\left(1.21 \times 10^{-6} \mathrm{~mol} \mathrm{~cm}^{-3}\right), D$ is the diffusion coefficient of the dissolved $\mathrm{O}_{2}\left(1.86 \times 10^{-5} \mathrm{~cm}^{2} \mathrm{~s}^{-1}\right)$, and $v$ is the kinetic viscosity of solvent $\left(0.01 \mathrm{~cm}^{2} \mathrm{~s}^{-1}\right) . \omega$ stands for the rotation rate of RDE in unit of rpm [30]. All the values are valid for $0.1 \mathrm{M} \mathrm{KOH}$ at $25^{\circ} \mathrm{C}$.

The yield of hydrogen peroxide $\left(\mathrm{H}_{2} \mathrm{O}_{2} \%\right)$ and apparent number of electron transferred during ORR $(n)$ were analyzed by using the RRDE measurements. The measurements were carried out by LSV from $0.2 \mathrm{~V}$ to $1 \mathrm{~V}$ vs. RHE at a scan rate of 5 $\mathrm{mV} \mathrm{s}^{-1}$ at $1600 \mathrm{rpm}$, and the ring electrode voltage was kept at $1.5 \mathrm{~V}$ vs. RHE. The percentage of peroxide released $\left(\mathrm{H}_{2} \mathrm{O}_{2} \%\right)$ and the apparent electron transfer number $(n)$ could be calculated by equations (2) and (3) [31].

$$
\begin{aligned}
& n=\frac{4 J_{\mathrm{D}}}{J_{\mathrm{D}}+\left(J_{\mathrm{R}} / N\right)} \\
& \mathrm{H}_{2} \mathrm{O}_{2} \%=100 \frac{2\left(J_{\mathrm{R}} / N\right)}{J_{\mathrm{D}}+\left(J_{\mathrm{R}} / N\right)}
\end{aligned}
$$

where the $\mathrm{H}_{2} \mathrm{O}_{2}$ collection coefficient $(N)$ at the ring is 0.37 .

EIS was carried out at a bias potential of $1.55 \mathrm{~V}$ vs. RHE and in a frequency range from 0.1 to $10^{6} \mathrm{~Hz}$. ECSA was derived using the in situ method based on the measurement of electrochemical double-layer capacitance $\left(C_{\mathrm{dl}}\right)$ in the non-Faradaic potential region. Specifically, CV measurements were performed in a potential window of $1.05-1.15 \mathrm{~V}$ vs. RHE at different scan rates of $10,15,20,25$, and $30 \mathrm{mV} \mathrm{s}^{-1}$. A plot of the charging current density, $J_{c}$ at $1.125 \mathrm{~V}$ vs. RHE, against the scan rates gives a straight line with a slope equal to $C_{\mathrm{dl}}$. Then the ECSA could be calculated based on Equation (4):

$$
E C S A=C_{\mathrm{dl}} / C_{S}
$$

Where $C_{\mathrm{s}}=60$ is the specific capacitance of the sample or the capacitance of an atomonically smooth planar surface with unit area under identical electrolyte conditions.

Assembly of the rechargeable Zn-air battery. The Zn-air battery was assembled using a zinc plate as the anode, $\mathrm{CoFe} / 3 \mathrm{D}-\mathrm{NLG}$ or a mixture of $\mathrm{Pt} / \mathrm{C}+\mathrm{RuO}_{2}$ loaded on carbon paper $\left(1 \mathrm{mg} \mathrm{cm}^{-2}\right)$ as the air cathode, and a solution of $6.0 \mathrm{M}$

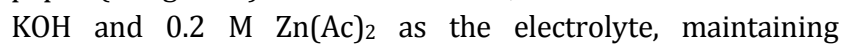
$\mathrm{O}_{2}$-saturated electrolyte throughout the test process. The $\mathrm{CHI}$ $600 \mathrm{E}$ electrochemistry workstation was adopted to test the battery discharge/charge performance and cycling stability.

\section{Results and discussion}

\subsection{Characterization and analysis}

Figure 1a displays the synthetic scheme for the CoFe/3D-NLG catalyst (see details in the Supporting Information). Firstly, 2D graphene oxide with in-plane mesopores (LGO) is produced by laser irradiation of graphene oxide sheets, which is then subjected to a hydrothermal nitrogen-doping and self-assembly process to acquire a 3D macro-/mesoporous graphene framework enriched with pyri-N (3D-NLG). Finally, $\mathrm{CoFe}_{2} \mathrm{O}_{4}$ NPs are grown on the surface of 3D-NLG to obtain a 3D macro-/mesoporous graphene framework enriched with pyri-N-Co active sites (CoFe/3D-NLG). Specifically, due to laser irradiation, the LGO sheets are markedly smaller in size than the initial graphene oxide (GO) sheets (Fig. S2a and S2b, Supporting Information), and they possess numerous mesopores created within the graphene planes (Fig. S2c and S2d, Supporting Information). Their decreased dimensions would be beneficial for avoiding agglomeration and extensive stacking of graphene sheets during the subsequent self-assembly process. Moreover, the numerous in-plane mesopores would provide additional edge sites to accommodate pyridinic nitrogen in the ensuing nitrogen-doping process, according to our previous study [14]. As the control sample, the nonirradiated GO sheets were utilized for the construction of a 3D N-doped graphene framework (3D-NG), according to an analogous procedure as for 3D-NLG. Expectedly, 3D-NLG (Fig. $1 \mathrm{~b}$ and Fig. S3b, Supporting Information) exhibits a superior cross-linked macroporous structure compared to 3D-NG (Fig. S3a, Supporting Information), suggesting that the smaller graphene sheets form the 3D cross-linked macroporous structure via self-assembly more readily, probably owing to the hydrophobicity of GO and $\pi-\pi$ stacking interactions [32].

$\mathrm{CoFe}_{2} \mathrm{O}_{4} \mathrm{NPs}$ were then grown onto the 3D-NLG support to acquire the $\mathrm{CoFe} / 3 \mathrm{D}-\mathrm{NLG}$ composite catalyst. $\mathrm{CoFe}_{2} \mathrm{O}_{4}$ NPs were likewise grown on 3D-NG, 2D N-doped graphene (NG), and 2D N-doped laser-irradiated graphene (NLG) to obtain control samples CoFe/3D-LG, CoFe/NG, and CoFe/NLG, respectively. The transmission electron microscopy (TEM) image (Fig. 1c) of CoFe/3D-NLG indicates that the NPs are anchored evenly on the graphene sheets with an average particle size of $8.1 \mathrm{~nm}$ (Fig. S4a, Supporting Information). The selected area electron diffraction pattern (inset in Fig. 1c) is indexed well with the crystal planes of the cubic spinel structure of $\mathrm{CoFe}_{2} \mathrm{O}_{4}$. The high-resolution TEM image illustrates that the NPs are highly crystalline (Fig. 1d), and that the inter-planar spacings of 0.296 and $0.253 \mathrm{~nm}$ match well with the (220) and (311) crystal planes of the $\mathrm{CoFe}_{2} \mathrm{O}_{4}$ lattice, respectively [33]. Additionally, the $\mathrm{X}$-ray diffraction pattern (Fig. S5, Supporting Information) verifies the crystal structure of $\mathrm{CoFe}_{2} \mathrm{O}_{4}$ (PDF\#22-1086). The scanning transmission electron microscopy image and elemental mapping indicate that $\mathrm{Co}, \mathrm{Fe}$, and $\mathrm{O}$ were primarily localized on the NPs, while $\mathrm{C}$ and $\mathrm{N}$ were uniformly distributed over the graphene framework of CoFe/3D-NLG (Fig. S6, Supporting Information). In all the composite samples, the weight percentages of $\mathrm{CoFe}_{2} \mathrm{O}_{4}$ NPs were comparable, at approximately 22 wt $\%-24$ wt $\%$ (Table S1, Supporting Information), as determined by inductively coupled plasma mass spectroscopy. The degree of graphene oxide reduction is a key factor for improving the electrical conductivity, as it is governed chiefly by the recovery

of $\pi$-conjugation within the GO sheets upon hydrothermal reduction [34]. According to the Fourier-transform infrared spectra (Fig. S7, Supporting Information), nitrogen doping 

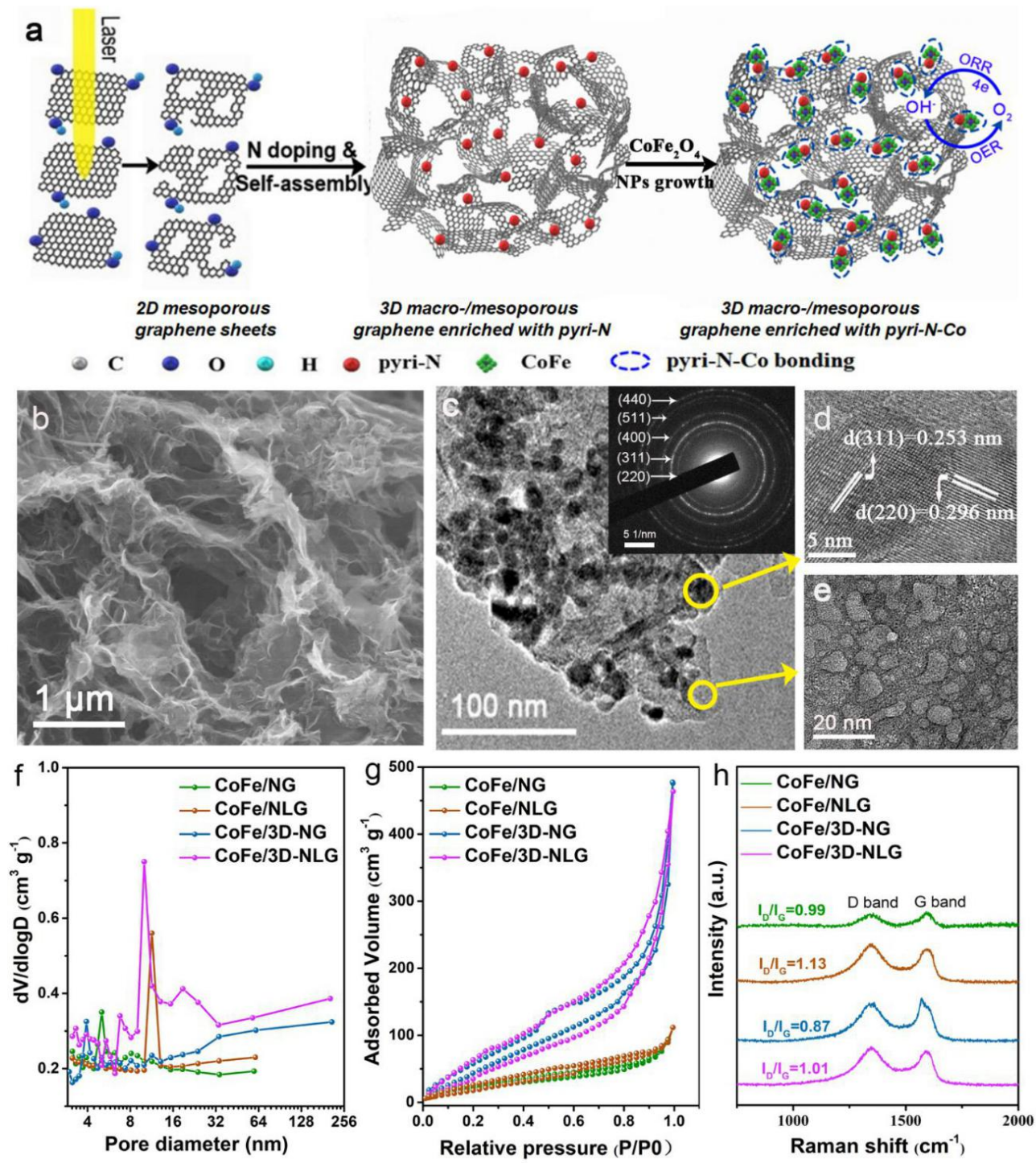

Fig. 1. (a) Synthetic scheme for the CoFe/3D-NLG catalyst. (b) SEM image of 3D-NLG. (c) TEM image of CoFe/3D-NLG. The inset shows the corresponding SAED pattern. (d) HR-TEM image of the NPs and (e) TEM image of the in-plane mesopores on the graphene sheet of CoFe/3D-NLG, as indicated by the yellow circles in c. (f) Pore size distribution, (g) $\mathrm{N}_{2}$ adsorption-desorption isotherm curves, and (h) Raman spectra of CoFe/NG, $\mathrm{CoFe} / \mathrm{NLG}, \mathrm{CoFe} / 3 \mathrm{D}-\mathrm{NG}$, and CoFe/3D-NLG.

resulted in the removal of a large number of oxygen-containing functional groups in 3D-NLG and CoFe/3D-NLG, suggesting the probability of enhanced electrical conductivity for these two catalysts. On the other hand, the enlarged TEM image (Fig. 1e) confirms the existence of numerous in-plane mesopores $(<20$ $\mathrm{nm}$ in diameter) in the graphene sheets of CoFe/3D-NLG. The overall pore size distribution of the samples was further quantitatively characterized using Brunauer-Emmett-Teller (BET) measurements. The pore size distribution curves (Fig. 1f) were derived from the adsorption isotherms based on the Barrett-Joyner-Halenda (BJH) model. The location of the peaks in Fig. 1f represents the concentrated distribution of pores in the composite catalysts. It is evident that the CoFe/3D-NLG catalyst possesses a hierarchically porous architecture, including both mesopores (8-20 nm) and macropores (> $100 \mathrm{~nm})$, in good agreement with the TEM images (Figs. $1 \mathrm{~b}$ and 1e). In contrast, $\mathrm{CoFe} / \mathrm{NLG}$ consists predominantly of mesopores, and
$\mathrm{CoFe} / 3 \mathrm{D}-\mathrm{NG}$ primarily contains macropores (Fig. 1f). The weak peaks corresponding to a pore size of 4-6 nm present in $\mathrm{CoFe} / \mathrm{NG}$ and $\mathrm{CoFe} / 3 \mathrm{D}-\mathrm{NG}$ were likely caused by holes formed between graphene sheets. Additionally, as seen in Fig. 1g, $\mathrm{CoFe} / 3 \mathrm{D}-\mathrm{NLG}$ has a large specific surface area $\left(322.6 \mathrm{~m}^{2} \mathrm{~g}^{-1}\right)$, which is more than four-fold larger than that of $2 \mathrm{D} \mathrm{CoFe} / \mathrm{NLG}$ $\left(78.6 \mathrm{~m}^{2} \mathrm{~g}^{-1}\right)$, confirming once more the porous structure of CoFe/3D-NLG. The control sample CoFe/3D-NG, which lacks in-plane mesopores, had a specific surface area $\left(348.4 \mathrm{~m}^{2} \mathrm{~g}^{-1}\right)$ comparable to that for $\mathrm{CoFe} / 3 \mathrm{D}-\mathrm{NLG}$, considering inevitable measurement errors, suggesting that the in-plane mesopores did not increase the surface area. Furthermore, the pore volume was likewise substantially augmented in CoFe/3D-NLG compared to its 2D counterpart (Table S2, Supporting Information). The laser-irradiation-created in-plane mesopore defects in graphene films were likewise evidenced by Raman spectroscopy, which provides direct evidence for graphene 
defects. As shown in Fig. 1h, the D and G bands correspond to defective and graphitic carbon structures, respectively. The intensity ratios of the $\mathrm{D}$ and $\mathrm{G}$ bands $\left(I_{\mathrm{D}} / I_{\mathrm{G}}\right)$ for CoFe/3D-NLG and $\mathrm{CoFe} / \mathrm{NLG}$ are higher than those for CoFe/3D-NG and $\mathrm{CoFe} / \mathrm{NG}$, implying that the laser-treated samples contain more defects in their graphene films [35], consistent with the observation of numerous in-plane graphene mesopores by TEM and BET measurements.

According to our previous studies, laser-induced in-plane mesopores preferentially benefit pyridinic nitrogen (in comparison to pyrrolic and graphitic nitrogen) doping into graphene sheets [14]. Electron energy loss spectroscopy (EELS) and X-ray photoelectron spectroscopy (XPS) were performed in order to analyze nitrogen configurations. As seen in the nitrogen K-edge EELS profiles (Fig. 2a), for CoFe/3D-NLG, the peak due to the pyridinic $\mathrm{N}$ (at $408.4 \mathrm{eV}$ ) has a higher intensity than that of the pyrrolic and graphitic $\mathrm{N}$ (at about $401 \mathrm{eV}$ ), in contrast to $\mathrm{CoFe} / \mathrm{NG}$, suggesting that more pyridinic $\mathrm{N}$ are present in $\mathrm{CoFe} / 3 \mathrm{D}-\mathrm{NLG}$ with a larger number of in-plane mesopores. Additionally, XPS survey data confirmed effective nitrogen doping of all the samples (Fig. S8 and Table S3, Supporting Information). High-resolution $\mathrm{N} 1 s$ spectra were recorded to acquire quantitative data on the distinct nitrogen configuration contents, which were deconvoluted into pyridinic N (398.4 eV), pyrrolic N (399.6 eV), and graphitic N (401.1 eV) (Fig. 2b and Fig. S9a and S9b Supporting Information) [36]. Expectedly, peaks relevant to the pyridinic $\mathrm{N}$ of the samples with in-plane mesopores (i.e., CoFe/3D-NLG, NLG, and CoFe/NLG) were more prominent than those with fewer mesopores (i.e., CoFe/NG, NG, and $\mathrm{CoFe} / 3 \mathrm{D}-\mathrm{NG}$ ). The contents of distinct nitrogen-doping configurations for all the samples are listed in Table S3 (Supporting Information). Specifically, the pyridinic $\mathrm{N}$ content for $\mathrm{CoFe} / 3 \mathrm{D}-\mathrm{NLG}$ was $3.07 \%$, which is significantly higher than that of $\mathrm{CoFe} / \mathrm{NG}$ (2.23\%), in good agreement with the EELS data. CoFe/NLG, the 2D counterpart of CoFe/3D-NLG, likewise exhibited a high pyridinic $\mathrm{N}$ content (3.02\%). Therefore, it was expected that abundant pyri-N-Co had formed in CoFe/3D-NLG and $\mathrm{CoFe} / \mathrm{NLG}$.

Next, the strong pyri-N-Co chemical bonding in CoFe/3D-NLG was evidenced by high-resolution XPS, EELS, and Raman spectroscopy. On one hand, the pyridinic and pyrrolic $\mathrm{N}$ 1s XPS peaks of CoFe/3D-NLG and CoFe/NG exhibit appreciable shifts toward a higher binding energy compared to that of 3D-NLG (Fig. 2b), implying that pyridinic and pyrrolic Ns are probably coordinated with the metal ions in CoFe/3D-NLG and $\mathrm{CoFe} / \mathrm{NG}$ [37]. Furthermore, the pyridinic $\mathrm{N}$ peak of CoFe/3D-NLG exhibits a further shift toward a higher binding energy relative to $\mathrm{CoFe} / \mathrm{NG}$, suggesting that a higher number of pyri-N-metal bonds exist in CoFe/3D-NLG. On the other hand, the Co $2 p_{3 / 2}$ XPS peak for CoFe/3D-NLG and CoFe/NG shift to a lower binding energy by $0.9 \mathrm{eV}$ and $0.6 \mathrm{eV}$ compared to $\mathrm{CoFe}_{2} \mathrm{O}_{4}$ (Fig. 2c), respectively. These XPS results suggest that Co is chemically coordinated with pyridinic and/or pyrrolic $\mathrm{N}$, resulting in partial electron transfer from Co to $\mathrm{N}$, and hence a higher oxidation state of Co ions in CoFe/3D-NLG and CoFe/NG [38]. The Co $2 p_{3 / 2}$ peak for CoFe/3D-NLG displayed a further shift toward a lower binding energy compared to CoFe/NG, indicating a higher degree of Co-N coordination in CoFe/3D-NLG. Meanwhile, the Co L-edge EELS peaks for CoFe/3D-NLG shift to higher energy loss region compared to CoFe/NG and $\mathrm{CoFe}_{2} \mathrm{O}_{4}$ (Fig. 2d), owing to a higher number of Co coordinated with N, consistent with the XPS results. Contrarily, both $\mathrm{Fe} 2 p_{3 / 2}$ XPS and Fe L-edge EELS profiles display indistinguishable peak shifts for CoFe/3D-NLG and CoFe/NG compared to $\mathrm{CoFe}_{2} \mathrm{O}_{4}$ (Fig. S10, Supporting Information), suggesting that Fe ions are not coordinated with $\mathrm{N}$. This may be because the
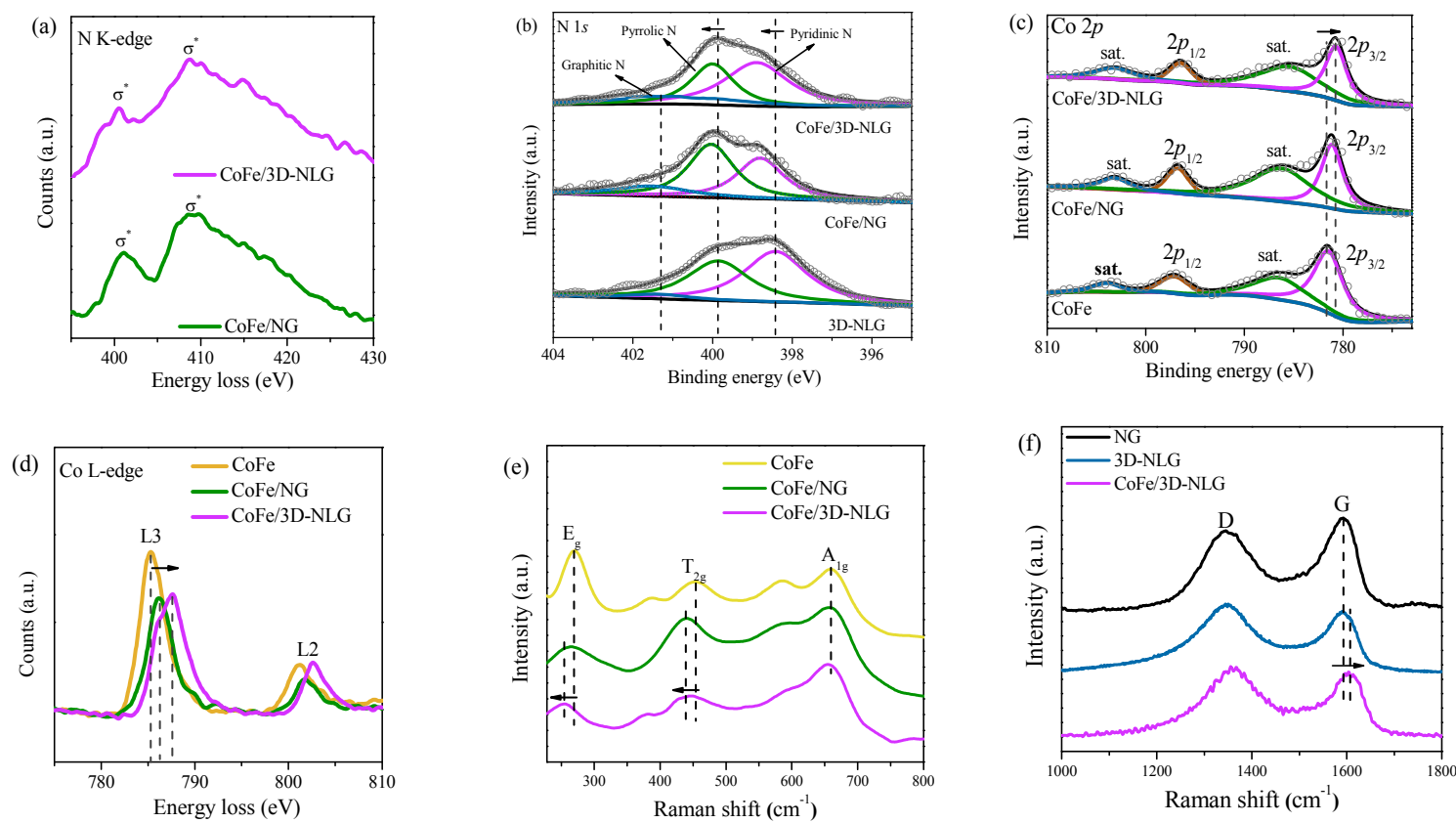

Fig. 2. (a) N K-edge EELS profiles of CoFe/NG and CoFe/3D-NLG. (b) N 1s XPS profiles of CoFe/3D-NLG, CoFe/NG, and 3D-NLG. (c) Co 2p XPS profiles, (d) Co L-edge EELS profiles, and (e) Raman spectra of CoFe, CoFe/NG, and CoFe/3D-NLG. (f) Raman spectra of NG, 3D-NLG, and CoFe/3D-NLG. 
Co- $\mathrm{N}_{X}$ moieties are formed more readily in graphene sheets than the $\mathrm{Fe}-\mathrm{N}_{x}$ moieties at the annealing temperature $\left(180{ }^{\circ} \mathrm{C}\right)$ adopted for $\mathrm{CoFe}_{2} \mathrm{O}_{4}$ NPs growth, which is too low to form chemical bonds between $\mathrm{Fe}$ and $\mathrm{N}$ [39-41]. In addition, the $\mathrm{C} 1 \mathrm{~s}$ spectrum of CoFe/3D-NLG was deconvoluted into $\mathrm{C}=\mathrm{C}, \mathrm{C}-\mathrm{O}$, and C-N, similar to that of 3D-NLG (Fig. S11, Supporting Information), whereas Fe-C and Co-C were not observed. Collectively, the above observations strongly imply that the $\mathrm{CoFe} / 3 \mathrm{D}-\mathrm{NLG}$ electrocatalyst possesses pyri-N-Co and pyrrolic-N-Co bonds. The pyri-N-Co bonds are expected to play a crucial role in enhancing the ORR/OER intrinsic activities, according to our previous findings [14]. The intense chemical interaction between $\mathrm{CoFe}$ NPs and nitrogen-doped graphene in $\mathrm{CoFe} / 3 \mathrm{D}-\mathrm{NLG}$ is also supported by the Raman spectra. Raman active modes for spinel $\mathrm{CoFe}_{2} \mathrm{O}_{4}$ are attributed to the motion of oxygen ions located at the octahedral (B) and tetrahedral (A) sites [42]. In Fig. 2e, the peak position at $660 \mathrm{~cm}^{-1}$ is assigned to a metal-oxygen respiration vibration in the tetrahedron $\left(\mathrm{AO}_{4}\right)$, and the other low-frequency vibration modes are related to the octahedron $\left(\mathrm{BO}_{6}\right)$. $\mathrm{CoFe}_{2} \mathrm{O}_{4}$ belongs to an "inverse" spinel structure, in which half of $\mathrm{Fe}$, not Co will preferentially occupy the center of the tetrahedron, and the center of the octahedron is occupied by the remaining half of $\mathrm{Fe}$ and Co [43]. The low-frequency peaks presented a red shift for CoFe/3D-NLG, which is caused by the weakened breath vibration of metal-oxygen, probably resulting from the strong chemical interaction between Co and N. Additionally, a blue shift was observed for the $\mathrm{G}$ band of CoFe/3D-NLG compared to the spectrum of 3D-NLG, which manifests from electron transfer occurring between CoFe NPs and nitrogen-doped graphene [44]. Consequently, based on the XPS, EELS, and Raman spectroscopy results, it can be concluded that a large number of pyri-N-Co bonds are formed in CoFe/3D-NLG, inducing effective electron transfer from Co to pyridinic N, which would appreciably enhance the intrinsic ORR/OER activity, as demonstrated below. Important to note is that compared to the monometal oxide, $\mathrm{Co}_{3} \mathrm{O}_{4}$, the introduction of $\mathrm{Fe}^{3+}$ ions into the $\mathrm{Co}_{3} \mathrm{O}_{4}$ network causes delocalization of the Co $3 \mathrm{~d}$ electrons and spin-state transition. $\mathrm{Fe}^{3+}$ ions can effectively activate adjacent $\mathrm{Co}^{3+}$ ions under the action of both spin and charge effects, resulting in enhanced intrinsic oxygen catalytic activity of the binary metal CoFe-based spinel oxide [45].

\subsection{The catalytic performance for OER}

The electrochemical performance was further explored, starting with the evaluation of OER activity by linear sweep voltammetry in $\mathrm{O}_{2}$-saturated $1 \mathrm{M} \mathrm{KOH}$ solution at a scan rate of $5 \mathrm{mV} \mathrm{s}^{-1}$ at room temperature. CoFe/3D-NLG exhibited substantially enhanced OER activity compared to that of 3D-NLG (Fig. S12a, Supporting Information), suggesting that the pyri-N-Co sites are significantly more active than $\mathrm{N}-\mathrm{C}$. As seen in Fig. 3a, CoFe/3D-NLG demonstrated the lowest overpotential of $304 \mathrm{mV}$ at a current density of $10 \mathrm{~mA} \mathrm{~cm}-2$, superior to CoFe/NLG (338 mV), CoFe/3D-NG (355 mV), and CoFe/NG $\left(390 \mathrm{mV}\right.$ ), surpassing the benchmark noble-metal $\mathrm{RuO}_{2}$ catalyst (322 mV). To better understand the excellent OER activity, reaction kinetics were then analyzed. As seen in Fig. $3 b$, the CoFe/3D-NLG catalyst exhibits the smallest Tafel slope, signifying enhanced kinetics for electrocatalytic oxygen evolution [46]. Notably, CoFe/3D-NLG and CoFe/NLG with numerous pyri-N-Co active sites have smaller Tafel slopes compared to CoFe/3D-NG and CoFe/NG, verifying that pyri-N-Co is beneficial for enhanced OER kinetics. In addition, although CoFe/3D-NLG and CoFe/NLG contain comparable amounts of pyri-N-Co, the Tafel slope of CoFe/3D-NLG is even smaller than that of $\mathrm{CoFe} / \mathrm{NLG}$, which is ascribed to the improved electrical conductivity of the 3D interconnected graphene architecture. The favorable kinetics were additionally confirmed by the electrochemical impedance spectra (EIS) (Fig. 3c), which provide information on charge transfer resistance [47]. A smaller semicircular arc corresponds to a lower charge transfer resistance $\left(R_{\mathrm{ct}}\right)$. Therefore, the CoFe/3D-NLG catalyst has the lowest $R_{\mathrm{ct}}$ (Table S4, Supporting Information), and the variation trend for all samples correlates with the Tafel slope data, suggesting once more that the pyri-N-Co active sites and 3D interconnected graphene framework could remarkably reduce $R_{\mathrm{ct}}$ and accelerate electron transfer, in turn promoting the OER kinetics. Furthermore, the electrochemical double-layer capacitance $\left(C_{\mathrm{dl}}\right)$ in the non-polarization region (Fig. 3d and Fig. S13 in Supporting Information) were measured to evaluate the electrochemical active surface area (ECSA), which is positively correlated to the density of accessible active sites [48]. The derived ECSA values are displayed in Fig. 3e, and evidently the ECSA of 3D porous catalysts, i.e., CoFe/3D-NLG and CoFe/3D-NG, are markedly higher than their 2D counterparts (CoFe/NLG and $\mathrm{CoFe} / \mathrm{NG}$ ), illustrating that a higher number of active sites are exposed due to the interlayer macropores present in CoFe/3D-NLG and CoFe/3D-NG. More importantly, the in-plane mesopores in CoFe/3D-NLG result in an additional increase of ECSA relative to CoFe/3D-NG, signifying that CoFe/3D-NLG possesses more active sites, likely due to the higher content of pyri-N-Co bonds in CoFe/3D-NLG compared to CoFe/3D-NG. As a measure of OER intrinsic activity, specific activity was calculated by normalizing the OER current at $1.6 \mathrm{~V}$ versus the reversible hydrogen electrode (RHE) with respect to the corresponding ECSA. As depicted in Fig. 3e, the specific activity of CoFe/3D-NLG displayed 3.6-fold and 1.7-fold enhancements relative to $\mathrm{CoFe} / 3 \mathrm{D}-\mathrm{NG}$ and $\mathrm{CoFe} / \mathrm{NG}$, respectively, implying that the pyri-N-Co active sites indeed promote the intrinsic catalytic activity for electrocatalytic oxygen evolution, consistent with our previous findings [14]. The above electrochemical analyses confirm that the enhanced OER performance for $\mathrm{CoFe} / 3 \mathrm{D}-\mathrm{NLG}$ is attributable to the combination of highly efficient pyri-N-Co active sites and the 3D porous graphene support with abundant in-plane mesopores and interlayer macropores. The former ensures high OER intrinsic activity, while the latter provides sufficient exposure and accessibility of the active sites to the reagents. Additionally, CoFe/3D-NLG manifests excellent long-term stability in $1.0 \mathrm{M} \mathrm{KOH}$ electrolyte after more than $15 \mathrm{~h}$ of continuous polarization, whereas the commercial $\mathrm{RuO}_{2}$ catalyst exhibits markedly inferior stability with a significant increase in the overpotential at a current density of $10 \mathrm{~mA} \mathrm{~cm}^{-2}$ after $15 \mathrm{~h}$ of operation (Fig. 3f). The su- 

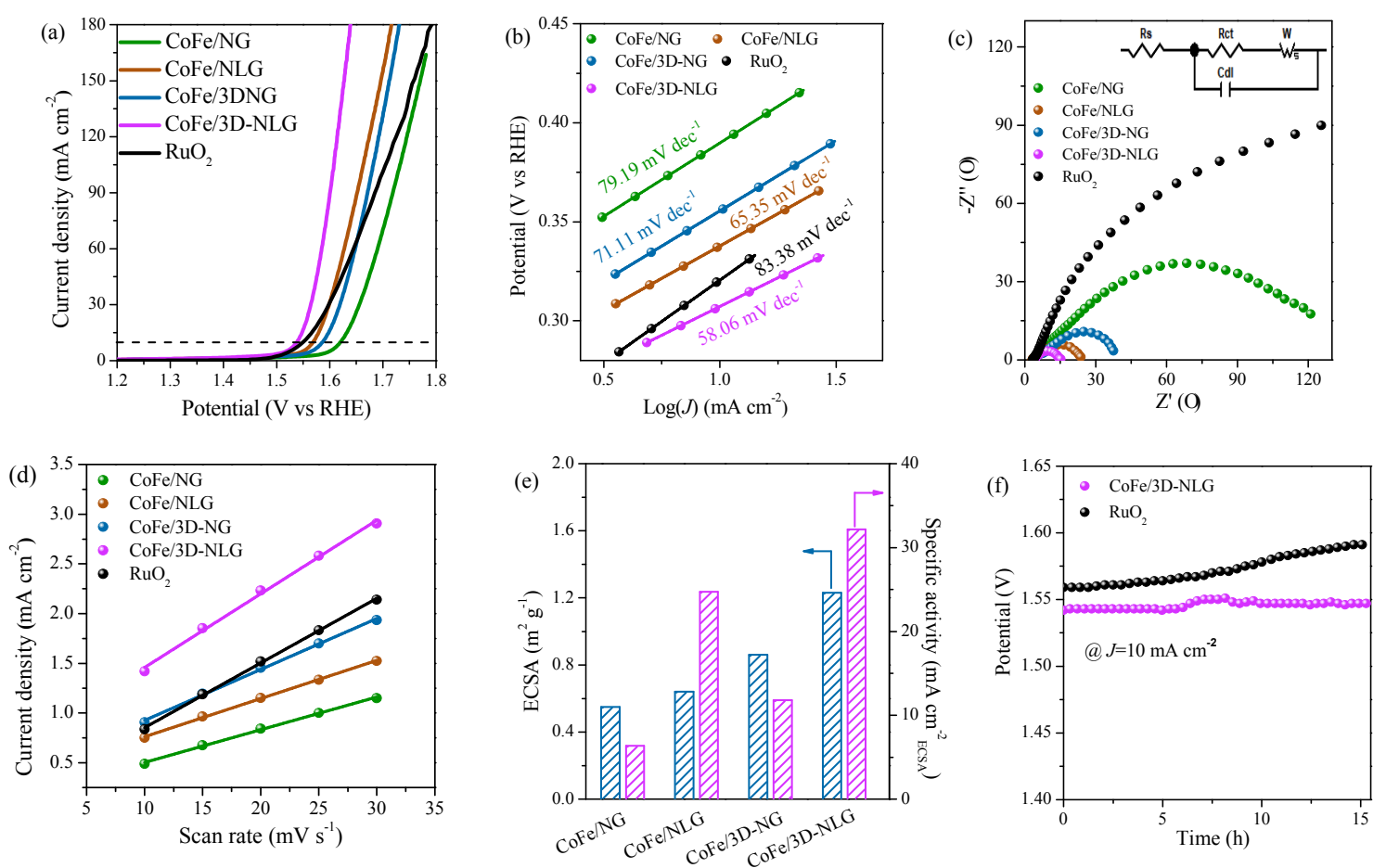

Fig. 3. (a) OER LSV curves, (b) Tafel plots, and (c) Nyquist plots of CoFe/NG, CoFe/NLG, CoFe/3D-NG, CoFe/3D-NLG, and RuO2. The inset in (c) shows the equivalent circuit used to calculate charge transfer resistance of the catalysts, where $R_{\mathrm{s}}$ is the electrolyte resistance, $R_{\mathrm{ct}}$ is the charge transfer resistance, and $C_{\mathrm{dl}}$ represents the double-layer capacitance. (d) Charging current density plots with varying scan rates for the samples. (e) Comparison of the ECSA and OER specific activity of different samples. (f) Chronoamperometric response (i- $t$ ) curves of CoFe/3D-NLG and RuO ${ }_{2}$.

perior durability of CoFe/3D-NLG arises from the strong chemical interaction between oxide NPs and the graphene support as revealed by XPS, EELS, and Raman spectroscopy, as well as from the stable 3D hierarchically porous graphene framework.

\subsection{The catalytic performance for ORR}

The advantageous microstructure features further endow $\mathrm{CoFe} / 3 \mathrm{D}-\mathrm{NLG}$ with excellent ORR performance. The ORR electrocatalytic behaviors of all the samples were firstly explored by cyclic voltammetry (CV) measurements in $0.1 \mathrm{M} \mathrm{KOH}$ solution. The higher ORR activity of CoFe/3D-NLG compared to that of 3D-NLG (Fig. S12b, Supporting Information) implies superior activity of pyri-N-Co compared to N-C. Fig. 4a displays the ORR LSV curves for all the catalysts. CoFe/3D-NLG and CoFe/NLG with abundant pyri-N-Co active sites have a higher onset potential ( $\left.E_{\text {onset }}\right)$ and half-wave potential $\left(E_{1 / 2}\right)$ than that of $\mathrm{CoFe} / 3 \mathrm{D}-\mathrm{NG}$ and CoFe/NG (Fig. 4b), implying that pyri-N-Co is responsible for the improved ORR kinetics. Owing to the highly conductive 3D hierarchically macro-/mesoporous structure of CoFe/3D-NLG, its ORR activity is further enhanced [13], with $E_{\text {onset }}(0.958 \mathrm{~V}$ vs. RHE) approaching that of the benchmark Pt/C catalyst (1.02 V vs. RHE), and $E_{1 / 2}(0.872 \mathrm{~V}$ vs. RHE) equivalent to Pt/C (0.876 V vs. RHE). The diffusion-limited current density of CoFe/3D-NLG exceeds that of Pt/C under a higher overpotential (Fig. 4a), due to rapid mass transfer within the 3D porous architecture [49]. The enhanced ORR kinetics are additionally corroborated by the small Tafel slope for CoFe/3D-NLG (46.33 $\mathrm{mV} \mathrm{dec}^{-1}$ ), which is comparable to that seen for Pt/C
(44.25 mV dec ${ }^{-1}$ ) (Fig. 4c). Additionally, the yield of hydrogen peroxide $\left(\mathrm{HO}_{2}^{-}\right)$and the apparent number of electrons transferred $(n)$ were obtained by adopting a rotating ring-disk electrode (RRDE) method. For CoFe/3D-NLG, $n$ is 3.85-3.95 (Fig. $\mathrm{S} 14$, Supporting Information), and the yield of $\mathrm{HO}_{2}-$ does not exceed $5 \%$ in the low overpotential region, indicating a nearly four-electron ORR pathway with a low $\mathrm{HO}_{2}$ - yield [50]. The yield increase of $\mathrm{HO}_{2}^{-}$in the high overpotential range is due to the involvement of additional two-electron transfer processes. Moreover, the ORR kinetic parameters were calculated using the Koutechy-Levich (K-L) plots derived from the rotating disk electrode (RDE) data (Figures S15a-e, Supporting Information). The slope of K-L plots (Figures S15f, Supporting Information) gives rise to an electron transfer number of 3.92 for CoFe/3D-NLG, akin to Pt/C (3.98), in agreement with the RRDE results. The kinetic current density $\left(\mathrm{V}_{\mathrm{k}, \mathrm{ORR}}\right)$ with mass transport correction was likewise derived from the RDE data. As shown in Fig. S16 (Supporting Information), CoFe/3D-NLG featuring numerous pyri-N-Co combined with a 3D macro-/mesoporous graphene structure exhibits a higher than two-fold enhancement in $J_{\mathrm{k}, \mathrm{ORR}}$ compared to $\mathrm{CoFe} / \mathrm{NG}$, outperforming Pt/C. Meanwhile, 2D CoFe/NLG with pyri-N-Co and CoFe/3D-NG and a 3D macroporous graphene structure resulted in approximately less than a one-fold increase in $J_{\mathrm{k}, \mathrm{ORR}}$ compared to $\mathrm{CoFe} / \mathrm{NG}$. As a measure of ORR intrinsic activity, mass activity was also calculated by normalizing $J_{\mathrm{k}, \mathrm{ORR}}$ at $0.7 \mathrm{~V}$ vs. RHE (Fig. S16, Supporting Information), with respect to the catalyst mass. As seen in Fig. 4d, the CoFe/3D-NLG catalyst demonstrated remarkably improved ORR mass activity, being 2.15 times 

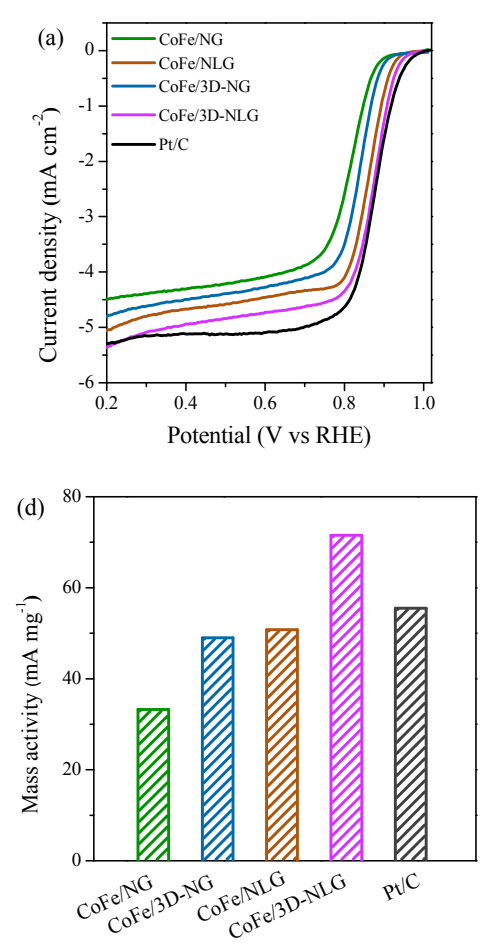
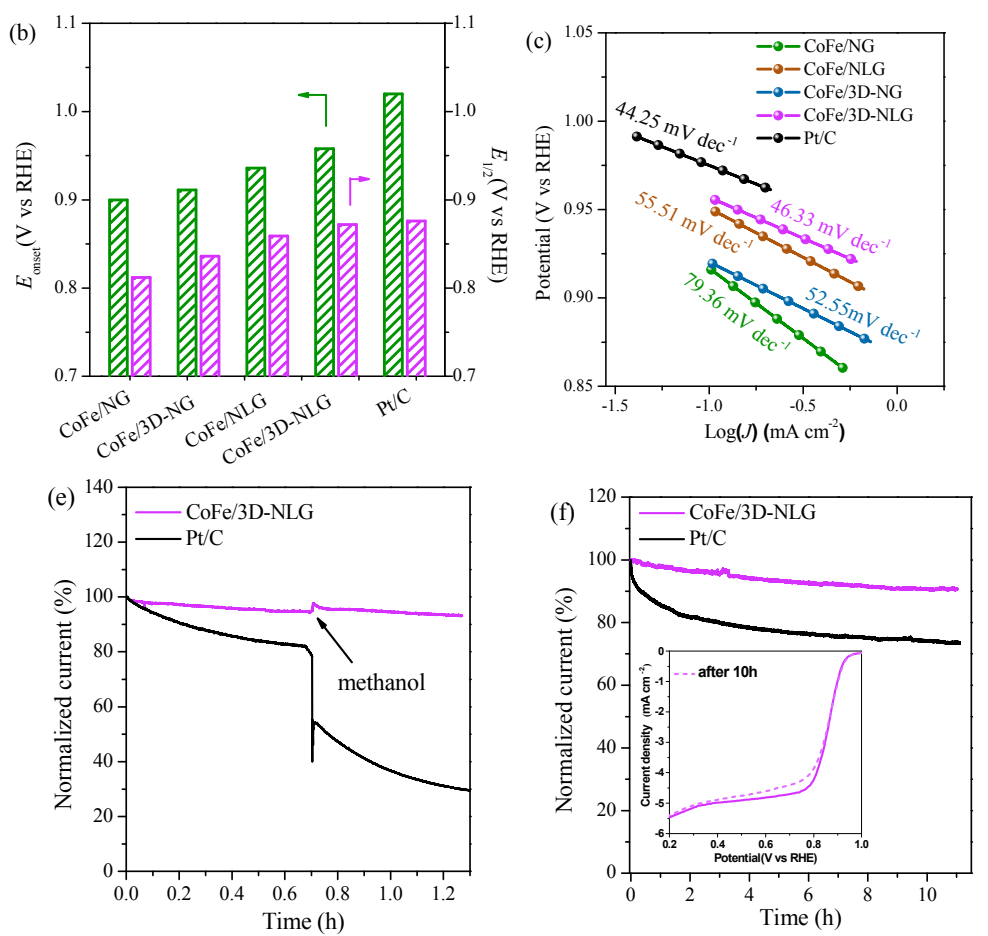

Fig. 4. (a) ORR LSV curves measured in $\mathrm{O}_{2}$-saturated $0.1 \mathrm{M} \mathrm{KOH}$ solution (rotation rate: 1600 rpm). (b) Corresponding bar plots of onset potential $\left(E_{\text {onset }}\right)$ and half-wave potential $\left(E_{1 / 2}\right)$. (c) Tafel plots. (d) Comparison of the ORR mass activity at $0.7 \mathrm{~V}$ vs. RHE. (e) $i$ - $t$ curve before and after the addition of $1.0 \mathrm{M}$ methanol. (f) Chronoamperometric response of CoFe/3D-NLG and Pt/C at $0.7 \mathrm{~V}$ vs. RHE. The inset shows the ORR LSV curves before and after $10 \mathrm{~h}$ of continuous polarization.

higher than that of $\mathrm{CoFe} / \mathrm{NG}$ and $28 \%$ higher relative to that of $\mathrm{Pt} / \mathrm{C}$. Consequently, the above results strongly indicate that for the CoFe/3D-NLG catalyst, the combination of copious pyri-N-Co active sites and 3D porous graphene composed of both in-plane mesopores and interlayer macropores does not only accelerate the ORR kinetics, but also facilitates rapid mass transfer, resulting in a substantial improvement in ORR activity [51]. In view of practical applications, a crossover effect was also examined. As seen in Fig. 4e, the CoFe/3D-NLG catalyst exhibits a significantly higher resistance to methanol crossover than does $\mathrm{Pt} / \mathrm{C}$, as no obvious ORR current alteration was observed for CoFe/3D-NLG upon injection of $1.0 \mathrm{M}$ methanol into the electrolyte. In addition to superior ORR activity, CoFe/3D-NLG exhibited superior long-term stability compared to Pt/C. Remarkably, for CoFe/3D-NLG, the ORR current density sustains $91 \%$ of the initial value after $10 \mathrm{~h}$ of continuous polarization, being exceedingly more stable than $\mathrm{Pt} / \mathrm{C}$, which exhibits a more severe current degradation (Fig. 4f). Furthermore, the polarization curve for CoFe/3D-NLG measured after $10 \mathrm{~h}$ displays negligible variation (inset of Fig. $4 \mathrm{f}$ ).

\subsection{Rechargeable zinc-air battery performance}

Based on the above electrochemical measurements, it can be concluded that the CoFe/3D-NLG catalyst exhibits excellent reversible ORR/OER electrocatalytic performance. As summarized in Fig. 5a, the ORR activity of CoFe/3D-NLG is comparable to that of the benchmark bifunctional noble-metal-based catalyst Pt/C $+\mathrm{RuO}_{2}$, while the OER activity surpasses that of $\mathrm{Pt} / \mathrm{C}+$
$\mathrm{RuO}_{2}$. Generally, the overall oxygen electrocatalytic activity can be quantitatively evaluated by the potential difference between ORR and OER, i.e., $\Delta E=E_{10}-E_{1 / 2}$, where $E_{10}$ denotes the potential required to deliver an OER current density of $10 \mathrm{~mA} \mathrm{~cm}{ }^{-2}$, and $E_{1 / 2}$ is the ORR half-wave potential. The $\Delta E$ value of an adequately efficient oxygen cathode for rechargeable zinc-air batteries should be as small as possible [52].

Remarkably, $\Delta E$ of CoFe/3D-NLG reached $0.67 \mathrm{~V}$, which is lower than that of $\mathrm{Pt} / \mathrm{C}+\mathrm{RuO}_{2}(0.69 \mathrm{~V})$ and superior to the majority of recently reported highly efficient Co- and/or Fe-based ORR/OER bifunctional catalysts (Table S5, Supporting Information). As the CoFe/3D-NLG catalyst exhibited superior ORR/OER activities and excellent long-term stability, its practical application in a self-manufactured rechargeable Zn-air battery was examined. As the oxygen cathode catalyst, CoFe/3D-NLG enables an exceedingly high battery open-circuit voltage of $1.56 \mathrm{~V}$, significantly higher than that of $\mathrm{Pt} / \mathrm{C}+\mathrm{RuO}_{2}$ (1.53 V) (Fig. 5b). The charge/discharge polarization curves demonstrate that the battery loaded with CoFe/3D-NLG has a higher voltaic efficiency compared to $\mathrm{Pt} / \mathrm{C}+\mathrm{RuO}_{2}$, as less voltage variation is required for $\mathrm{CoFe} / 3 \mathrm{D}-\mathrm{NLG}$ to achieve a high current density in the charge/discharge process (Fig. 5c). The peak power density attains $213 \mathrm{~mW} \mathrm{~cm}^{-2}$ for $\mathrm{CoFe} / 3 \mathrm{D}-\mathrm{NLG}$, outperforming Pt/C $+\mathrm{RuO}_{2}$ (190 $\mathrm{mW} \mathrm{cm}^{-2}$ ) (Fig. 5c), being one of the highest values among recently reported highly efficient Co- and/or Fe-based ORR/OER bifunctional catalysts (Table S5, Supporting Information). The specific discharge capacity for CoFe/3D-NLG achieves $456.5 \mathrm{~mA} \mathrm{~h} \mathrm{~g} \mathrm{zn}^{-1}$, by normalization to the mass of consumed zinc, which is markedly larger than that 

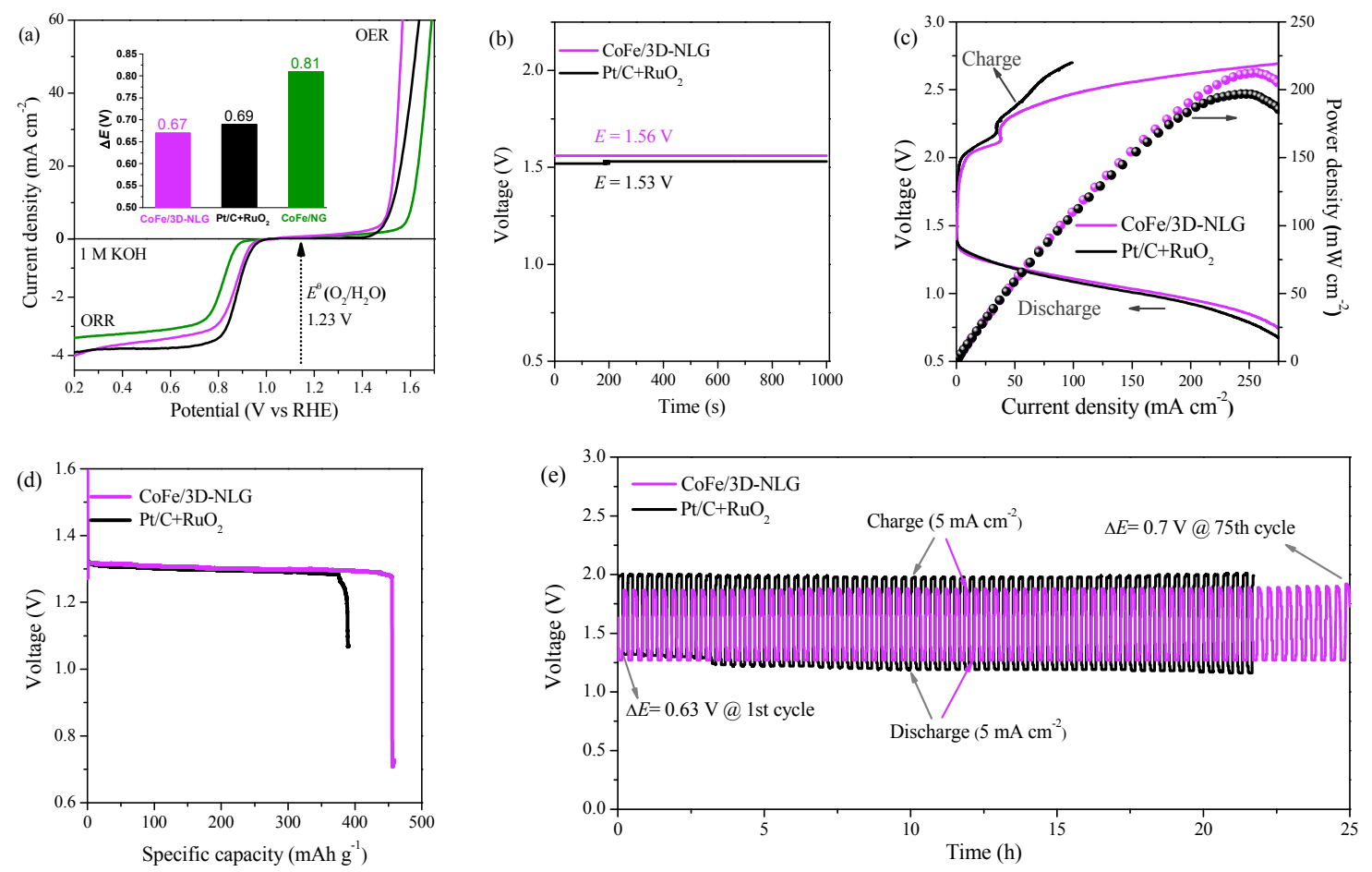

Fig. 5. (a) The overall polarization curves of CoFe/3D-NLG, CoFe/NG, and Pt/C $+\mathrm{RuO}_{2}$, measured in $\mathrm{O}_{2}$-saturated $1.0 \mathrm{M} \mathrm{KOH}$ solution. The inset shows the potential difference between ORR and OER $(\Delta E)$ for the catalysts. (b) Open-circuit plots, (c) galvanodynamic charge/discharge profiles and power density curves, (d) discharge curves at a constant current density of $10 \mathrm{~mA} \mathrm{~cm}^{-2}$, and (e) charge/discharge cycling curves at a current density of $5 \mathrm{~mA}$ $\mathrm{cm}^{-2}$ of Zinc-air batteries assembled with $\mathrm{CoFe} / 3 \mathrm{D}-\mathrm{NLG}$ and $\mathrm{Pt} / \mathrm{C}+\mathrm{RuO}_{2}$ (duration: $1200 \mathrm{~s}$ per cycle).

of Pt/C + RuO2 (391.2 mA h g $\mathrm{zn}^{-1}$ ) (Fig. 5d). Additionally, Fig. 5e demonstrates the charge/discharge cycling curves of $\mathrm{Zn}$-air batteries. The $\mathrm{CoFe} / 3 \mathrm{D}-\mathrm{NLG}$ catalyst requires an ultra-low charge/discharge voltage $(0.63 \mathrm{~V})$, lower than $\mathrm{Pt} / \mathrm{C}+\mathrm{RuO}_{2}$ requires $(0.69 \mathrm{~V})$. After cycling charge/discharge at $5 \mathrm{~mA} \mathrm{~cm}^{-2}$ for 75 cycles $(\approx 25 \mathrm{~h}$ ), a mere $11 \%$ increase in the overpotential was observed, in sharp contrast to $\mathrm{Pt} / \mathrm{C}+\mathrm{RuO}_{2}$, which exhibits a more drastic overpotential increment. The above tests confirm once more the excellent reversible ORR/OER performance of the CoFe/3D-NLG electrocatalyst relative to state-of-the-art noble-metal catalysts, as well as its feasibility as an oxygen electrode catalyst in rechargeable Zn-air batteries.

\section{Conclusions}

In conclusion, a 3D hierarchically porous graphene framework composed of interconnected in-plane mesopores and interlayer macropores enriched with pyri-N-Co active sites, namely CoFe/3D-NLG, was assembled and applied as a highly efficient reversible ORR/OER electrocatalyst. The ORR onset potential and half-wave potential attained $0.96 \mathrm{~V}$ and $0.87 \mathrm{~V}$ (vs. RHE), respectively, being comparable to the benchmark $\mathrm{Pt} / \mathrm{C}$ catalyst. Additionally, both the ORR mass activity and long-term stability are considerably higher than observed for $\mathrm{Pt} / \mathrm{C}$. Furthermore, The OER overpotential at $10 \mathrm{~mA} \mathrm{~cm}-2$ attains a low value of $304 \mathrm{mV}$ for CoFe/3D-NLG, outperforming the noble-metal $\mathrm{RuO}_{2}$ catalyst. Finally, CoFe/3D-NLG achieves a very low potential difference between ORR and OER $(0.67 \mathrm{~V})$, being lower than that of $\mathrm{Pt} / \mathrm{C}+\mathrm{RuO}_{2}(0.69 \mathrm{~V})$, and is among the lowest values for Co- and/or Fe-based ORR/OER bifunctional electrocatalysts reported thus far. When applied as the oxygen electrode in a rechargeable Zn-air battery, CoFe/3D-NLG manifested excellent charge/discharge behavior, surpassing the bifunctional $\mathrm{Pt} / \mathrm{C}+\mathrm{RuO}_{2}$ catalyst. The enhanced ORR/OER activities of CoFe/3D-NLG are attributed to the followings: (i) The abundant pyri-N-Co active sites crucially accelerate the sluggish ORR/OER kinetics, leading to remarkably enhanced intrinsic activities per active site for oxygen electrocatalysis; (ii) The numerous in-plane mesopores within each graphene sheet render the interlayer macropores comprehensively interconnected, thus providing additional shortcuts for reagent and product diffusion; (iii) The 3D graphene structure with favorable conductivity proved advantageous for rapid charge transfer in catalytic processes. This work provides a new strategy for the construction of low-cost and efficient oxygen electrocatalysts with improved intrinsic activity and a higher proportion of exposed active sites for application in rechargeable metal-air batteries.

\section{References}

[1] M. Armand, J. M. Tarascon, Nature, 2008, 451, 652-657.

[2] L. Trotochaud, S. W. Boettcher, Scr. Mater., 2014, 74, 25-32.

[3] G. Fu, X. Yan, Y. Chen, L. Xu, D. Sun, J. M. Lee, Y. Tang, Adv. Mater., 2018, 30, 1704609/1-1704609/11.

[4] A. Morozan, B. Jousselme, S. Palacin, Energy Environ. Sci., 2011, 4, 1238-1254.

[5] Y. Fang, Y. Guo, Chin. J. Catal., 2018, 39, 566-582. 
[6] Q. Liu, L. Du, G. Fu, Z. Cui, Y. Li, D. Dang, X. Gao, Q. Zheng, J. B. Goodenough, Adv. Energy Mater., 2019, 9, 1803040/11803040/7.

[7] G. Ma, R. Jia, J. Zhao, Z. Wang, C. Song, S. Jia, Z. Zhu, J. Phys. Chem. C, 2011, 115, 25148-25154.

[8] J. Jin, J. Yin, H. Liu, P. Xi, Chin. J. Catal., 2019, 40, 43-51.

[9] X. Wen, X. Yang, M. Li, L. Bai, J. Guan, Electrochim. Acta, 2019, 296, 830-841.

[10] W. Yan, X. Cao, J. Tian, C. Jin, K. Ke, R. Yang, Carbon, 2016, 99, 195-202.

[11] C. Zhang, J. Liu, Y. Ye, Z. Aslam, R. Brydson, C. Liang, ACS Appl. Mater. Interfaces, 2018, 10, 2423-2429.

[12] S. Sun, G. Shen, J. Jiang, W. Mi, X. Liu, L. Pan, X. Zhang, J. J. Zou, Adv. Energy Mater., 2019, 9, 1901505/1-1901505/8.

[13] Z. S. Wu, S. Yang, Y. Sun, K. Parvez, X. Feng, K. Mullen, J. Am. Chem. Soc. 2012, 134, 9082-9085.

[14] X. R. Wang, J. Y. Liu, Z. W. Liu, W. C. Wang, J. Luo, X. P. Han, X. W. Du, S. Z. Qiao, J. Yang, Adv. Mater., 2018, 30, 1800005.

[15] H. Miao, S. Li, Z. Wang, S. Sun, M. Kuang, Z. Liu, J. Yuan, Int. J. Hydrog. Energy, 2017, 42, 28298-28308.

[16] Y. Li, J. Yang, J. Huang, Y. Zhou, K. Xu, N. Zhao, X. Cheng, Carbon, 2017, 122, 237-246.

[17] D. A. Dikin, S. Stankovich, E. J. Zimney, R. D. Piner, G. H. B. Dommett, G. Evmenenko, S. T. Nguyen, R. S. Ruoff, Nature, 2007, 448, 457-460.

[18] J. He, M. Wang, W. Wang, R. Miao, W. Zhong, S.-Y. Chen, S. Poges, T. Jafari, W. Song, J. Liu, S. L. Suib, ACS Appl. Mater. Interfaces, 2017, 9, 42676-42687.

[19] F. Shi, X. Zhu, W. Yang, Chin. J. Catal., 2020, 41, 390-403.

[20] Y. Li, T. Gao, Y. Yao, Z. Liu, Y. Kuang, C. Chen, J. Song, S. Xu, E. M. Hitz, B. Liu, R. J. Jacob, M. R. Zachariah, G. Wang, L. Hu, Adv. Energy Mater., 2018, 8, 1801289/1-1801289/8.

[21] L. Xiao, Q. Yang, M. J. Wang, Z. X. Mao, J. Li, Z. Wei, J. Mater. Sci.,
2018, 53, 15246-15256.

[22] X. Li, Y. Zhao, Y. Yang, S. Gao, Nano Energy, 2019, 62, 628-637.

[23] Z. Li, Q. Gao, X. Liang, H. Zhang, H. Xiao, P. Xu, Z. Liu, Carbon, 2019, 150, 93-100.

[24] S. Chen, S. Z. Qiao, ACS Nano, 2013, 7, 10190-10196.

[25] Z. Zuo, T. Y. Kim, I. Kholmanov, H. Li, H. Chou, Y. Li, Small, 2015, 11, 4922-4930.

[26] Y. Chai, Z. Li, J. Wang, Z. Mo, S. Yang, J. Alloys Compd., 2019, 775, 1206-1212.

[27] M. Mi, X. Liu, W. Kong, Y. Ge, W. Dang, J. Hu, Desalination, 2019, $464,18-24$.

[28] W. S. Hummers, R. E. Offeman, J. Am. Chem. Soc., 1958, 80, 1339-1339.

[29] W. Lv, C. Zhang, Z. Li, Q. H. Yang, J. Phys. Chem Lett., 2015, 6, 658-668.

[30] J. Du, Y. Pan, T. Zhang, X. Han, F. Cheng, J. Chen, J. Mater. Chem. A, 2012, 22, 15812-15818.

[31] Y. Liang, H. Wang, J. Zhou, Y. Li, J. Wang, T. Regier, H. Dai, J. Am. Chem. Soc., 2012, 134, 3517-3523.

[32] G. Wang, L.-T. Jia, B. Hou, D.-B. Li, J.-G. Wang, Y.-H. Sun, New Carbon Mater., 2015, 30, 30-40.

[33] M. Li, Y. Xiong, X. Liu, X. Bo, Y. Zhang, C. Han, L. Guo, Nanoscale, 2015, 7, 8920-8930.

[34] D. Kong, W. Yuan, C. Li, J. Song, A. Xie, Y. Shen, Appl. Surf. Sci., 2017, 393, 144-150.

[35] G. Lin, R. Ma, Y. Zhou, Q. Liu, X. Dong, J. Wang, Electrochim. Acta, 2018, 261, 49-57.

[36] Z. Huang, Z. Liao, W. Yang, H. Zhou, C. Fu, Y. Gong, L. Chen, Y. Kuang, Electrochim. Acta, 2017, 245, 957-966.

[37] X. Cui, S. Yang, X. Yan, J. Leng, S. Shuang, P. M. Ajayan, Z. Zhang, Adv. Funct. Mater. 2016, 26, 5708-5717.

[38] M. Zeng, Y. Liu, F. Zhao, K. Nie, N. Han, X. Wang, W. Huang, X. Song, J. Zhong, Y. Li, Adv. Funct. Mater., 2016, 26, 4397-4404.

\section{Graphical Abstract}

Chin. J. Catal., 2021, 42: 571-582 doi: 10.1016/S1872-2067(20)63642-7

3D hierarchically macro-/mesoporous graphene frameworks enriched with pyridinic-nitrogen-cobalt active sites as efficient reversible oxygen electrocatalysts for rechargeable zinc-air batteries

Sheng Zhou, Jiayi Qin, Xueru Zhao, Jing Yang *

Tianjin University

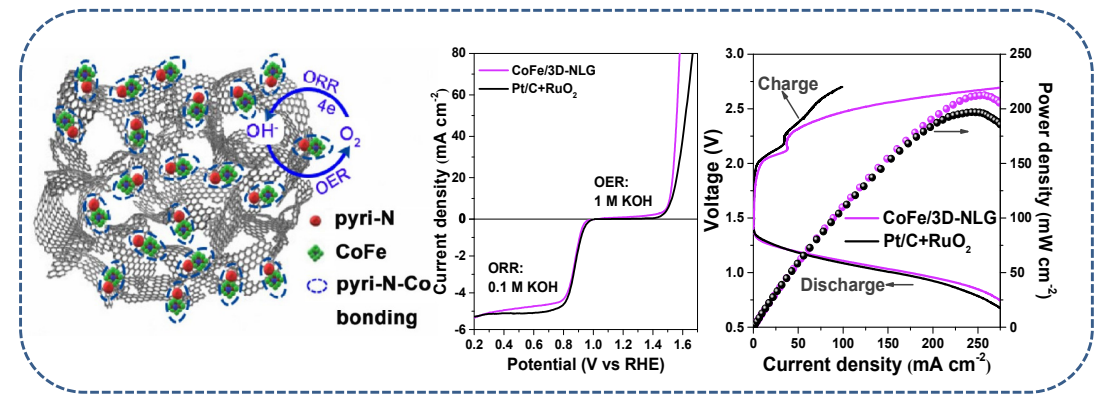

An efficient reversible oxygen electrocatalyst consisting of 3D hierarchically macro-/mesoporous graphene frameworks with abundant pyridinic-nitrogen-cobalt active sites is prepared. It exhibits low overpotentials for both ORR and OER and excellent performance in Zn-air batteries. 
[39] X. Fu, Y. Liu, X. Cao, J. Jin, Q. Liu, J. Zhang, Appl. Catal. B, 2013, 130-131, 143-151.

[40] W. I. Choi, S.-H. Jhi, K. Kim, Y.-H. Kim, Phys. Rev. B, 2010, 81, 085441/1-085441/5.

[41] J. M. Ziegelbauer, T. S. Olson, S. Pylypenko, F. Alamgir, C. Jaye, P. Atanassov, S. Mukerjee, J. Phys. Chem. C, 2008, 112, 8839-8849.

[42] Y. Li, Y. Li, Y. Yin, D. Xia, H. Ding, C. Ding, J. Wu, Y. Yan, Y. Liu, N. Chen, P. K. Wong, A. Lu, Appl. Catal. B, 2018, 226, 324-336.

[43] Y. Kumar, A. Sharma, P. M. Shirage, J. Alloys Compd., 2019, 778, 398-409.

[44] L. Wei, H. E. Karahan, S. Zhai, H. Liu, X. Chen, Z. Zhou, Y. Lei, Z. Liu, Y. Chen, Adv. Mater., 2017, 29, 1701410/1-1701410/11.

[45] X. T. Wang, T. Ouyang, L. Wang, J. H. Zhong, T. Ma, Z. Q. Liu, Angew. Chem. Int. Ed., 2019, 58, 13291-13296.
[46] X. Liu, M. Park, M. G. Kim, S. Gupta, G. Wu, J. Cho, Angew. Chem. Int. Ed., 2015, 54, 9654-9658.

[47] Y. Meng, W. Song, H. Huang, Z. Ren, S. Y. Chen, S. L. Suib, J. Am. Chem. Soc., 2014, 136, 11452-11464.

[48] J. Zhang, L. Dai, ACS Catal., 2015, 5, 7244-7253.

[49] X. Tong, S. Chen, C. Guo, X. Xia, X. Y. Guo, ACS Appl. Mater. Interfaces, 2016, 8, 28274-28282.

[50] Y. Liang, H. Wang, J. Zhou, Y. Li, J. Wang, T. Regier, H. Dai, J. Am. Chem. Soc., 2012, 134, 3517-3523.

[51] Y. Shao, M. F. El-Kady, C. W. Lin, G. Zhu, K. L. Marsh, J. Y. Hwang, Q. Zhang, Y. Li, H. Wang, R. B. Kaner, Adv. Mater., 2016, 28, 6719-6726.

[52] M. Wu, G. Zhang, J. Qiao, N. Chen, W. Chen, S. Sun, Nano Energy, 2019, 61, 86-95.

\title{
富含吡啶氮-钴活性位点的三维多级大/介孔石墨烯作为高效可逆氧电催化剂 用于可充电锌-空气电池
}

\author{
周 省, 覃佳艺, 赵雪茹, 杨 静* \\ 天津大学材料科学与工程学院新能源材料研究所, 先进陶瓷与加工技术教育部重点实验室, 天津 300072
}

\begin{abstract}
摘要: 随着能源危机的日益严峻, 能源的储存和转换越来越受到人们的重视. 目前人们加以开发和利用的清洁能源主要包 括太阳能、风能、氢能、地热能以及电化学能等. 其中, 燃料电池和金属-空气电池等作为电化学器件为电化学能的开发及 可持续利用提供了条件. 特别是金属-空气电池以电极电位较负的金属如镁、铝、锌、铁等作负极, 以空气中的氧或纯氧作 正极, 具有比能量高、性能稳定、价格便宜的特点. 氧还原反应(ORR)和析氧反应(OER)是可再生电化学能量转换和储存过 程中的两个关键电化学过程. 贵金属 $\left(\mathrm{Pt} / \mathrm{C}, \mathrm{Ir} / \mathrm{C}, \mathrm{IrO}_{2}\right.$ 等)虽然具有高催化活性, 但价格昂贵、资源贵乏限制了其大规模的使 用和发展. 此外, 它们的催化性能单一, 难以同时实现多反应的高效催化. 目前, 大量研究工作集中在开发低成本、高效的 ORR 和OER催化剂, 用来代替昂贵的铂类贵金属催化剂. 在能源器件设计中, 由于OER和ORR反应发生在同一个电极上, 若能制备出具有ORR和OER双功能催化性能的电催化剂, 将在很大程度上降低能源器件的设计难度. 最近, 我们的研究工 作揭示了吡啶-氮-钴(pyri-N-Co)配位结构在协同作用中的重要性, 协同作用大幅度提升了 $\mathrm{NiCo}_{2} \mathrm{O}_{4} / \mathrm{N}$ 掺杂石墨烯的本征催 化活性. 虽然金属粒子与掺氮石墨烯的结合有利于催化活性和稳定性的提高, 但二维石墨烯片之间由于 $\pi-\pi$ 键相互作用, 容 易聚集和堆叠. 在实际应用中, 石墨烯片之间的堆叠会导致可达表面的损失, 从而使复合催化剂利用率降低, 结构稳定性 变差. 因此,制备富含充分暴露且高效的ORR/OER活性中心的电催化剂仍然是一个巨大挑战.

本文采用激光辐照法和水热法制备了具有层间大孔和片内介孔相互交联结构且负载铁酸钴纳米颗粒的三维多级孔石 墨烯复合电催化剂( $\mathrm{CoFe} / 3 \mathrm{D}-\mathrm{NLG})$, 研究了其微观结构与ORR/OER电催化性能的关系. 比表面积和X射线光电子能谱测试 结果表明, CoFe/3D-NLG 具有大的比表面积 $\left(322.6 \mathrm{~m}^{2} \mathrm{~g}^{-1}\right)$ 和孔体积 $\left(0.715 \mathrm{~cm}^{3} \mathrm{~g}^{-1}\right)$, 并且富含吡啶氮-钴活性中心. 电化学测 试表明, 对于 $O E R$ 电催化, $\mathrm{CoFe} / 3 \mathrm{D}-\mathrm{NLG}$ 复合催化剂在 $10 \mathrm{~mA} \mathrm{~cm}$ 处的过电势为 $304 \mathrm{mV}$, 优于商用 $\mathrm{RuO}_{2}$ 催化剂的 $322 \mathrm{mV}$; 对于 ORR电催化, $\mathrm{CoFe} / 3 \mathrm{D}-\mathrm{NLG}$ 的半波电位达到 $872 \mathrm{mV}$, 非常接近商用 $\mathrm{Pt} / \mathrm{C}$ 催化剂 $(876 \mathrm{mV})$. 此外, 作为可充电锌空气电 池的空气电极催化剂, CoFe/3D-NLG展现出了超高的开路电压 $(1.56 \mathrm{~V})$ 、高功率密度 $\left(213 \mathrm{~mW} \mathrm{~cm}^{-2}\right)$ 以及超低充放电电压 $(0.63 \mathrm{~V})$, 并且具有良好的充放电循环稳定性. CoFe/3D-NLG优异的ORR/OER电催化性能主要归因于以下两点：1)大量的 吡啶氮-钴活性位点极大地加快了缓慢的氧电催化动力学, 提高了每个活性位点的ORR/OER本征催化活性; 2)丰富的层间 大孔和面内介孔多级孔结构促进了整个石墨烯结构中的高效传质, 因而在电催化过程中吡啶氮-钴活性位点得以充分暴露 于电解液中.
\end{abstract}

关键词: 多级孔; 复合催化剂; 氧电催化; 尖晶石氧化物; 可充电锌-空气电池

收稿日期: 2020-04-27. 接受日期: 2020-05-31. 上网日期: 2020-09-05.

*通讯联系人. 电话: (022)85356663; 电子信箱: yang jing@tju.edu.cn

基金来源：国家自然科学基金(51572188, 51822106).

本文的电子版全文由Elsevier出版社在ScienceDirect上出版(http://www.sciencedirect.com/science/journal/18722067). 